\title{
Study of the Effect of the Minimum Acquisition Price Policy on Food Security
}

\author{
Xiaoqian Liu, a , Qi Yin ${ }^{2, b}$, Chang'an Wang ${ }^{3, c}$ \\ ${ }^{1,2}$ School of Management, Sichuan Agricultural University, Chengdu 611130, China \\ ${ }^{3}$ Research Institute of Economics and Management, Southwestern University Of Finance And \\ Economics, Chengdu 611130, China
}

\begin{abstract}
Keywords: The minimum acquisition price policy;food security;fix-effect model.
Abstract. The minimum acquisition price policy for grain plays an important role in food production. Research on the reasonable minimum grain acquisition price has an important strategic significance for the stable development of agriculture in our country. This paper establishes the evaluation of minimum grain acquisition price policy implementation effect model. Consider grain varieties and regional differences, this paper chooses five major grain producing provinces to compare the effect of implementation of the minimum acquisition price policy. The empirical study shows that grain purchasing price and grain acreage have a positive and significant effect on grain yield. Agricultural subsidy policy, which can effectively encourage farmers to increase grain acreage.
\end{abstract}

\section{Introduction}

Grain is not only necessary food in people's daily life, but also strategic materials for maintain national economic development and political stability. With the decrease of arable land, the increase of population, shortage of water resources, climate changes and other issues having become increasingly prominent, besides the international grain market impacts, China's grain industry faces potential risk. Therefore, it is very important to study food protection policy in China. Seeing the evaluation results from the policy, Luping Lan(2013) considers the minimum acquisition price policy distort the market pricing rules, because in some parts the acreage of some varieties falls instead of rising, the policy effect is suspect.Under the implement of the minimum acquisition price in China, Junjun Ren, Wenju Wang(2010) use market Supply-Demand model to study the influence of it on Supply-Demand Curve. Some scholars believe that there is a lack in China's minimum acquisition price policy and put forward some improvement methods. Such as Hong Fang(2009) suggests compensation method can be used to the appropriate elements of grain production. Fusheng Zeng (2011) believes that minimum acquisition price policy has a significantly benefit to grain output and farmers income, which can improve the enthusiasm of farmers to grow more. Guangyan Yang (2006) and Shihai Wang (2011) believes that the effect of China minimum acquisition price policy on grain yield is significant, which is conducive to improving the enthusiasm of farmers, Mingxing Chen (2007) believes that in addition to analysis policy effect on increasing grain yield and income, but also we should consider the environmental cost of policy implementation and consider the comprehesive effect of the policy.

This article chooses 2005 to 2014 as time series, choose five regions which implement the minimum perchase price policy as cross-sectional data, including Hunan, Hubei, Jiangxi, Anhui and Sichuan five provinces, to study the influence of minimum acquisition policy mainly on grain yield.

\section{VARIBLE DATA SOURCES And MODEL CONSTRCTION}

Variable selection. Regard food production as food security(explained variable), this paper studies the influence of lowest acquisition policy mainly on grain yield (i.e. food security) .Investigate the current minimum acquisition policy effect on grain yield. Choose the price of agricultural means of production (production cost), grain acquisition price (minimum acquisition price) and grain acreage area as explanatory variables. It can be seen from the economic significance, the effect of the price of 
agricultural means of production on grain yield is negative, the effect of the grain acquisition price (minimum acquisition price) and grain acreage on the grain yield is positive.

Sample selection and data sources. In 2004, the minimum acquisition price policy came into effect in China, ,this article chooses 2005 to 2014 as time series, choose five regions which implement the minimum acquisition price policy as cross-sectional data rice minimum, including Hunan, Hubei, Jiangxi, Anhui and Sichuan five provinces. Because in the rice producing areas, grain minimum acquisition policy has a large influence on the grain yield, so it has a representitive siginificance. The data of grain yield, grain acreage area and agricultural means of production price are from the China Statistical Yearbook, the grain acquisition price data is from the State Grain Administration and the National Statistics Bureau website.

\section{Theoretical Model Construction}

Farmer behavior model. Taking the types of agricultural subsidy policy implemented in different regions of china into account:

$$
\begin{aligned}
& M a x \pi=\left(\mathrm{p}_{1}+\theta_{1}\right) \mathrm{q}_{1}+\mathrm{p}_{2} \mathrm{q}_{2}+\theta_{2} N_{1}+\theta_{3} \mathrm{q}_{1}-\left[w_{1}\left(L_{1}+L_{2}\right)+w_{2}\left(N_{1}+N_{2}\right)+w_{3}\left(K_{1}+K_{2}\right)\right] \\
& \text { s.t. } \mathrm{q}_{1}=A_{1} L_{1}^{\alpha_{1}} N_{1}^{\beta_{1}} K_{1}^{\gamma_{1}}, 0<\alpha_{1}+\beta_{1}+\gamma_{1}=\sigma_{1}<1 \\
& \mathrm{q}_{2}=A_{2} L_{2}^{\alpha_{2}} N_{2}^{\beta_{2}} K_{2}^{\gamma_{2}}, 0<\alpha_{2}+\beta_{2}+\gamma_{2}=\sigma_{2}<1
\end{aligned}
$$

$\mathrm{p}_{1}, \mathrm{p}_{2}$ respectively represents the price of grain crops and economic crops, $\mathrm{q}_{1}, \mathrm{q}_{2}$ respectively represents crops and economic crops production, $L_{1}, L_{2}$ respectively represents the number of labor input on economic crops and grain crops, $N_{1}, N_{2}$ respectively represents the arable land input on grain crops and economic crops, $K_{1}, K_{2}$ respectively represents fertilizer, pesticides and other agricultural production materials input on grain crops and economic crops, ${ }^{w_{1}},{ }^{w_{2}},{ }^{w_{3}}$ respectively represents the number of labor, land, and other agricultural prices, $\theta_{1}$ is the part under the minimum acquisition price policy acquistion price higher than the market price, $\theta_{2}$ is direct subsidies according to the actual acreage per acre, $\theta_{3}$ is direct subsidies according to the practical amount soldper kilogram. $\theta_{1} \mathrm{q}_{1}$ is subsidies got by farmers under the minimum acquisition policy, $\theta_{2} N_{1}$ is direct subsidies got by farmers under the area, $\theta_{3} \mathrm{q}_{1}$ is subisidies got by farmers under trasaction. The optimal yield of grain and the optimal production factor input quantity equation can be obtained from the first order condition of profit maximization:

$$
\begin{aligned}
& \mathrm{q}_{1}^{*}=F_{1} \cdot\left(\frac{1}{w_{2}-\theta_{2}}\right)^{\frac{\beta_{1}}{1-\sigma_{1}}}\left(\mathrm{p}_{1}+\theta_{1}+\theta_{3}\right)^{\frac{\sigma_{1}}{1-\sigma_{1}}} \quad L_{1}^{*}=F_{1} \cdot \frac{\alpha_{1}}{w_{1}\left(w_{2}-\theta_{2}\right)^{\frac{\beta_{1}}{1-\sigma_{1}}}}\left(\mathrm{p}_{1}+\theta_{1}+\theta_{3}\right)^{\frac{1}{1-\sigma_{1}}} \\
& N_{1}^{*}=F_{1} \cdot \beta_{1}\left(\frac{1}{w_{2}-\theta_{2}}\right)^{\frac{1-\alpha_{1}-\gamma_{1}}{1-\sigma_{1}}}\left(\mathrm{p}_{1}+\theta_{1}+\theta_{3}\right)^{\frac{1}{1-\sigma_{1}}} K_{1}^{*}=F_{1} \cdot \frac{\gamma_{1}}{w_{3}\left(w_{2}-\theta_{2}\right)^{\frac{\beta_{2}}{1-\sigma_{1}}}}\left(\mathrm{p}_{1}+\theta_{1}+\theta_{3}\right)^{\frac{1}{1-\sigma_{1}}} \\
& F_{1}=\left[A_{1}\left(\frac{\alpha_{1}}{w_{1}}\right)^{\alpha_{1}}\left(\beta_{1}\right)^{\beta_{1}}\left(\frac{\gamma_{1}}{w_{3}}\right)^{\gamma_{1}}\right]^{\frac{1}{1-\sigma_{1}}}
\end{aligned}
$$

It can be seen from the the output equation $\mathrm{q}_{1}^{*}$ that the output of grain is affected by the factors such as grain price, the number of the minimum acquisition price, the number of direct subsidies and the price of factors of production.Similarly, we can get the optimal yield of economic crops and the optimal input of production factor quantity equation: 


$$
\begin{aligned}
\mathrm{q}_{2}^{*} & =F_{2} \cdot \mathrm{p}_{2}^{\frac{\sigma_{2}}{1-\sigma_{2}}} \quad L_{2}^{*}=F_{2} \cdot \frac{\alpha_{2}}{w_{1}} \mathrm{p}_{2}^{\frac{1}{1-\sigma_{2}}} \quad N_{2}^{*}=F_{2} \cdot \frac{\beta_{2}}{w_{2}} \mathrm{p}_{2}^{\frac{1}{1-\sigma_{2}}} \quad K_{2}^{*}=F_{2} \cdot \frac{\gamma_{2}}{w_{3}} \mathrm{p}_{2}^{\frac{1}{1-\sigma_{2}}} \\
F_{2} & =\left[A_{2}\left(\frac{\alpha_{2}}{w_{1}}\right)^{\alpha_{2}}\left(\frac{\beta_{2}}{w_{2}}\right)^{\beta_{2}}\left(\frac{\gamma_{2}}{w_{3}}\right)^{\gamma_{2}}\right]^{\frac{1}{1-\sigma_{2}}}
\end{aligned}
$$

The effect of the minimum acquisition price on grain yield. According to the above profit maximization equation, grain yield $\mathrm{q}_{1}^{*}$ is obtained, the effect of the minimum acquisition price policy, direct subsidies according to the actual planting area and the influence of direct subsidy according to the amount of sales on grain yield can be analyzed.In the analysis of the relationship between the minimum acquisition price policy and the grain yield, we take the derivatives of $\mathrm{q}_{1}^{*}$ to ${ }_{1}$, and the result is greater than zero.

$$
\frac{\partial \mathrm{q}_{1}^{*}}{\partial \theta_{1}}=F_{1} \cdot \frac{\sigma_{1}}{1-\sigma_{1}}\left(\frac{1}{w_{2}-\theta_{2}}\right)^{\frac{\beta_{1}}{1-\sigma_{1}}}\left(\mathrm{p}_{1}+\theta_{1}+\theta_{3}\right)^{\frac{2 \sigma_{1}-1}{1-\sigma_{1}}}>0
$$

In the analysis of the relationship between the direct subsidy policy in practical planting areas and grain production,we take the derivative of $\mathrm{q}_{1}^{*}$ to $\theta_{2}$, and the result is greater than zero, indicating that the implementation of direct subsidies according to the planting area can promote the increase of grain yield.

$$
\frac{\partial \mathrm{q}_{1}^{*}}{\partial \theta_{2}}=F_{1} \cdot \frac{\beta_{1}}{1-\sigma_{1}}\left(\frac{1}{w_{2}-\theta_{2}}\right)^{\frac{1-\alpha_{1}-\gamma_{1}}{1-\sigma_{1}}}\left(\mathrm{p}_{1}+\theta_{1}+\theta_{3}\right)^{\frac{\sigma_{1}}{1-\sigma_{1}}>0}
$$

In the analysis of the relationship between the number of transactions carried out according to the direct subsidy policy and grain yield,we take the derivative of $\mathrm{q}_{1}^{*}$ to $\theta_{3}$, and the result is greater than zero, indicating that the implementation of direct subsidies according to the trasaction can promote the increase of grain yield.

$\frac{\partial \mathrm{q}_{1}^{*}}{\partial \theta_{3}}=F_{1} \cdot \frac{\sigma_{1}}{1-\sigma_{1}}\left(\frac{1}{w_{2}-\theta_{2}}\right)^{\frac{\beta_{1}}{1-\sigma_{1}}}\left(\mathrm{p}_{1}+\theta_{1}+\theta_{3}\right)^{\frac{2 \sigma_{1}-1}{1-\sigma_{1}}}>0$

Therefore, the direct given subsidy policy according to the actual planting area and the number of transactions tas the standard implementation can promote the improvement of grain production, which helps to promote the realization of the goal of food security.

\section{Empirical Analysis}

This paper implement fixed-effected model, $Y_{\mathrm{it}}=\alpha_{\mathrm{i}}+X_{\mathrm{it}} \beta+U_{\mathrm{it}}, i=1,2,3,4,5$

$\mathrm{t}=2005,2006 \cdots 2014$, there are three explanatory variables, the $X_{\text {it }}(S 、 B 、 T)$ is $1 * 3$ vector, $S$ is on behalf of the acreage area, $B$ represents the cost of agricultural production, $T$ represents the minimum grain acquisition price, ${ }^{\alpha}$ is the individual effect, reflecting the impact of individual

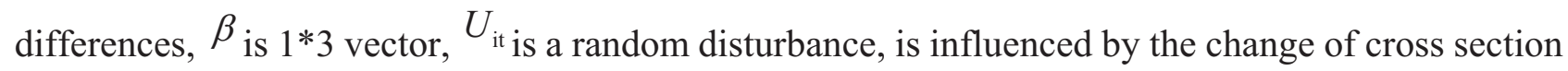
and time factors.Model results generated in the eviews 8.0 software, fixed coefficient effect model analysis is applied.Results are as follows: 
Table 1: Fixed effects model for assessing food safety outcomes

\begin{tabular}{ccccc}
\hline Variable & Coefficient & Std. & T-statistic & Prob. \\
\hline C & 3.9688 & 1.3290 & 2.9863 & 0.0047 \\
S & 0.6018 & 0.2407 & 2.5003 & 0.0164 \\
B & -0.0014 & 0.0008 & -1.6245 & 0.1117 \\
T & 0.2430 & 0.0785 & 3.0953 & 0.0035 \\
SICHUAN-C & 0.0085 & & $\mathrm{R}^{2}$ & 0.9853 \\
ANHUI-C & -0.1418 & & F-Statistic & 401.5101 \\
HUNAN-C & 0.1291 & & Prob(F-statistic) & 0.0000 \\
JIANGXI-C & -0.0441 & & Log likelihood & 110.0291 \\
HUBEI-C & 0.0483 & & D.W & 1.48 \\
\hline
\end{tabular}

The model operation results show that the regression coefficient of the acquisition price of grain and grain planting area are significant, while the price of agricultural means of production (production cost) although the sign is negative, consistent with its economic significance, but not significant; F value shows the model's linear relationship is very significant, adjusted $R^{2}$ can reach 0.9853 which shows that the model has a good goodness of fit; $D . W$ value is 1.48 , indicating that the model residuals have no serial correlation. The analysis results, Analyze from the overall, this model has a good effect. The results are analyzed as follows:

The material price (production cost price) on grain yield is negative and not significant. The direct reason is that price increase of agricultural means of production,means production costs rise. From a certain angle, which will make grain farmers reduce pesticides, fertilizer using amount, actually reducing production cost, which may effect the grain yield and quality. However, the price of agricultural means of production is not the main factors affecting the yield, to a certain extent, the price does not have a significant impact on grain yield, in other words:Even though the price of agriculture production is vey high, farmers will still grow grain.

Grain purchasing price and acreage area have a positive and significant effect on grain yield, fit real economic significance. From the point of farmers view, the grain acquisition price is food sales price, therefore, the higher price of grain yield has a stronger stimulation on production; from the grain sowing area, only ensure a certain amount of grain acreage area, national food safety can be fundamentally protected. Therefore, to a certain extent, the grain yield depends on the size of grain acreage area.

\section{Conclusions and recommendations}

Food safety is a priority in the target system of the grain minimum acquisition price policy, indicating that the grain acquisition price will be significant to it. From 2005 to 2014, seeing from the change of the lowest acquisition price, because of China's agricultural population accounts for the majority,considering the per capita arable land area and the actual planting area difference, the lowest acquisition policy not benefits a lot to each farmer. Therefore, the grain minimum acquisition policy cannot become significant factors affecting farmers' income. However, from the greatly changed coefficient of grain purchasing variables, we can see increase the grain acquisition price other than other variables might have play a more significant role, which provide empirical basis to the government implementati on minimum acquisition policy, and point a direction to grain minimum acquisition price policy: it is meaniful to continue improving the grain acquisition price level. We can further carry out agricultural subsidy policy, which can effectively encourage farmers to increase grain acreage and increase grain production intensive management level, to improve the level of grain production and then ensure national food security policy.

\section{References}

[1] Luping Lan,Problems and effects of China's grain minimum purchase price policy and proposals. Research of Agricultural Modernization, (5),pp. 3-7,2013. 
[2] Junjun Ren, Wenju Wang, Study on the development of the minimum purchase price policy in China. Journal of Hubei University of Economics (HUMANITIES AND SOCIAL SCIENCES EDITION), 07(6),pp. 29-30, 2010.

[3] Hong Fang,Study on the mechanism of the reasonable determination of the minimum acquisition price of grain in China . Economics and Management, (23),pp. 20-25,2009.

[4] Fusheng Zeng,Peng Dai. Analysis of the effect of increasing the production and income of grain price protection policy. Agricultural Economics and Management, (3),pp.11-17,2010.

[5] Guangyan Yang, Analysis of the effect of the minimum grain purchase price policy. Price Theory and Practice, (6),pp. 35-36,2006.

[6] Minxing Chen. Analysis of the effect of grain direct subsidy and policy implications. Journal of Shandong Agricultural University (SOCIAL SCIENCE EDITION), 9(1),pp.51-54,2007. 